\title{
Quiste de duplicación gástrico: diagnóstico por punción-aspiración guiada por ecoendoscopia
}

\author{
S. Seijo Ríos ${ }^{1,2}$, J. Lariño Noia ${ }^{1,2}$, I. Abdulkader Nallib ${ }^{3}$, A. Lozano León ${ }^{2}$, B. Vieites Pérez-Quintela ${ }^{3}$, \\ J. Iglesias García ${ }^{1,2}$ y J. E. Domínguez Muñoz ${ }^{1,2}$
}

${ }^{2}$ Servicio de Aparato Digestivo. ${ }^{2}$ Fundación para la Investigación en Enfermedades del Aparato Digestivo (FIENAD). ${ }^{3}$ Servicio de Anatomía Patológica. Hospital Clínico Universitario. Santiago de Compostela, A Coruña

\begin{abstract}
RESUMEN
Los quistes de duplicación son anomalías congénitas infrecuentes del tracto gastrointestinal que pueden localizarse a cualquier nivel desde la boca hasta al ano. Los quistes de duplicación gástricos son los más excepcionales y constituyen tan sólo el 4-8\% de todos ellos. Su patogénesis es controvertida y hoy en día aun existen múltiples teorías que tratan de explicar su mecanismo etiopatogénico. En la mayoría de los casos el diagnóstico se realiza en la primera infancia y tan sólo en una pequeña proporción se identifican en la edad adulta como un hallazgo incidental, ya que estas entidades suelen ser asintomáticas fuera de la infancia. Casi en la mitad de los casos se asocia a otras malformaciones congénitas y es de vital importancia realizar un minucioso diagnóstico diferencial con otras entidades, en especial neoplasias con componente quístico. Aunque es extremadamente inusual, se ha publicado la degeneración neoplásica de estas lesiones, lo que hace más importante su preciso diagnóstico y estudio citohistológico. En este artículo se presenta el caso de un quiste de duplicación gástrico diagnosticado en la edad adulta, mediante ultrasonografía endoscópica asociado a punción-aspirativa, técnica que ha sido recientemente propuesta como la más eficaz para el diagnóstico.
\end{abstract}

Palabras clave: Quiste duplicación gástrico. Ultrasonografía endoscópica (USE). Punción aspiración guiada por ultrasonografía endoscópica (USE-PAAF).

\begin{abstract}
Duplication cysts are rare gastrointestinal congenital abnormalities. They can occur anywhere throughout the gastrointestinal tract, and gastric duplication cysts are most uncommon, representing only $4-8 \%$ of all gastrointestinal duplication cysts. Nowadays several theories try to explain the pathogenic mechanisms involved. These cysts are usually diagnosed during early childhood, and very rarely are detected in adults, mostly incidentally due to a lack of symptoms. Close to $50 \%$ of gastric cysts are associated with other abnormalities. It is extremely important that a meticulous differential diagnosis is performed regarding other diseases, mainly malignancies with a cystic component. Although extremely uncommon, a malignant transformation of these lesions has been reported, which highlights the importance of a correct diagnosis. Herein we report the case of a duplication cyst in an adult, which was detected by endoscopic ultrasound-guided fineneedle aspiration, recently proposed as the most accurate technique for the identification of these lesions.
\end{abstract}

Key words: Gastric duplication cyst. Endoscopic ultrasound (EUS). EUS guided fine-needle aspiration (EUS-FNA).

Seijo Ríos S, Lariño Noia J, Abdulkader Nallib I, Lozano León A, Vieites Pérez-Quintela B, Iglesias García J, Domínguez Muñoz JE. Quiste de duplicación gástrico: diagnóstico por punción-aspiración guiada por ecoendoscopia. Rev Esp Enferm Dig 2008; 100: 586-590.

\section{INTRODUCCIÓN}

Los quistes de duplicación constituyen una entidad extremadamente infrecuente. Se trata de anomalías congé-

Recibido: 05-05-08.

Aceptado: 03-06-08.

Correspondencia: Susana Seijo Ríos. Servicio de Aparato Digestivo. Hospital Clínico Universitario. C/ Choupana, s/n. 15706 Santiago de Compostela. A Coruña. e-mail: susanaseijo@hotmail.com nitas de muy baja prevalencia que pueden localizarse a cualquier nivel del tracto gastrointestinal. De todos ellos, los localizados en el estómago y en el apéndice son los menos frecuentes.

El diagnóstico se realiza en más de la mitad de los casos en la primera infancia, ya que a esta edad suelen ser sintomáticos. Por el contario, en la edad adulta estos quistes suelen ser indolentes y su diagnóstico constituye un hallazgo incidental. En más del 50\% de los casos suelen asociarse a otras malformaciones, principalmente gastrointestinales y vertebrales, y en ocasiones pueden 
complicarse con hemorragias, la fistulización o incluso con trasformación maligna de su epitelio. Las técnicas de diagnóstico habitualmente empleadas como el TAC abdominal y la RMN, presentan múltiples dificultades para su caracterización y no son infrecuentes los errores diagnósticos con lesiones sólidas. Recientemente han sido publicados varios artículos que destacan la eficacia diagnóstica de la ecoendoscopia en la caracterización de estas entidades en comparación con las técnicas convencionales de imagen descritas.

Hoy en día aun no está establecido un algoritmo terapéutico. La cirugía está recomendada para los casos sintomáticos o en los que se haya producido una complicación, pero hasta el momento no existe consenso en cuanto a la actitud a seguir en los casos asintomáticos. Esto constituye un problema, sobre todo si se considera el riesgo de transformación maligna de estas lesiones.

A pesar de ser descrita ya en el 1911 por Wendel, desde entonces han sido muy escasos los casos publicados. Por todo ello se presenta el caso de un quiste de duplicación gástrico en un paciente de edad adulta diagnosticado por punción guiada por ecografía endoscópica y una posterior revisión de la literatura.

\section{CASO CLÍNICO}

Se trata de un varón de 39 años de edad remitido a nuestra Unidad de Ecoendoscopia para filiar la naturaleza de una lesión submucosa localizada en la vertiente gástrica de la unión esófago-gástrica. El paciente inicia su estudio médico en el Servicio de Otorrinolaringología donde consulta por molestias faríngeas. Tras las exploraciones llevadas a cabo por este servicio se decide la realización de una endoscopia digestiva alta, donde se evidencia, a $36 \mathrm{~cm}$ de arcada dentaria, una lesión redondeada, con mucosa conservada de un diámetro mayor aproximado de $1,5 \mathrm{~cm}$. Se toman biopsias mediante endoscopia, siendo el resultado histológico no concluyente. Ante la presencia de una lesión submucosa, cuya naturaleza no es posible filiar mediante la imagen endoscópica ni tras el estudio histológico de las biopsias se remite al paciente a nuestro centro para la realización de una ecoendoscopia (USE).

La USE se realiza de forma ambulatoria, bajo monitorización de constantes vitales y sedación consciente a base de midazolam y petidina. Se emplea un ecoendoscopio lineal Pentax EG3830UX acoplado a un ecógrafo Hitachi 8500 . A $36 \mathrm{~cm}$ de arcada dentaria se identifica una pequeña lesión anecoica, bien delimitada, de aspecto quístico, que parece depender de la submucosa, de 14 x 6 mm de diámetros máximos (Figs. 1 y 2). Mediante el estudio ultrasonográfico no se identifica la presencia de adenopatías abdominales ni mediastínicas y se demuestra la integridad del eje vascular. Una vez identificada la lesión a puncionar se realiza un estudio ecográfico con doppler color para evitar formaciones vasculares e identificar el trayecto más adecuado para realizar la punción. Para dicha punción se emplea la aguja Echotip Wilson-Cook de 19 G. En primer lugar se extrae la vaina de la aguja unos centímetros a través del canal de trabajo hasta ser visualizada bien gastroscópica- o ecoendoscópicamente; seguidamente se procede a puncionar la lesión mediante la aguja situada en el interior de la vaina. Una vez verificada su posición en el interior de la lesión, se retira el estilete (el cual se emplea para prevenir una posible contaminación de la muestra) y se realizan maniobras de avance de la aguja con el fin de extraer la mayor cantidad de material posible de la lesión. En nuestro caso hemos empleado una jeringa de aspiración para aumentar la celularidad de la muestra. Se realizan dos pases sobre la lesión hasta que el patólogo, presente en la sala de exploraciones, confirma la suficiencia de la muestra.

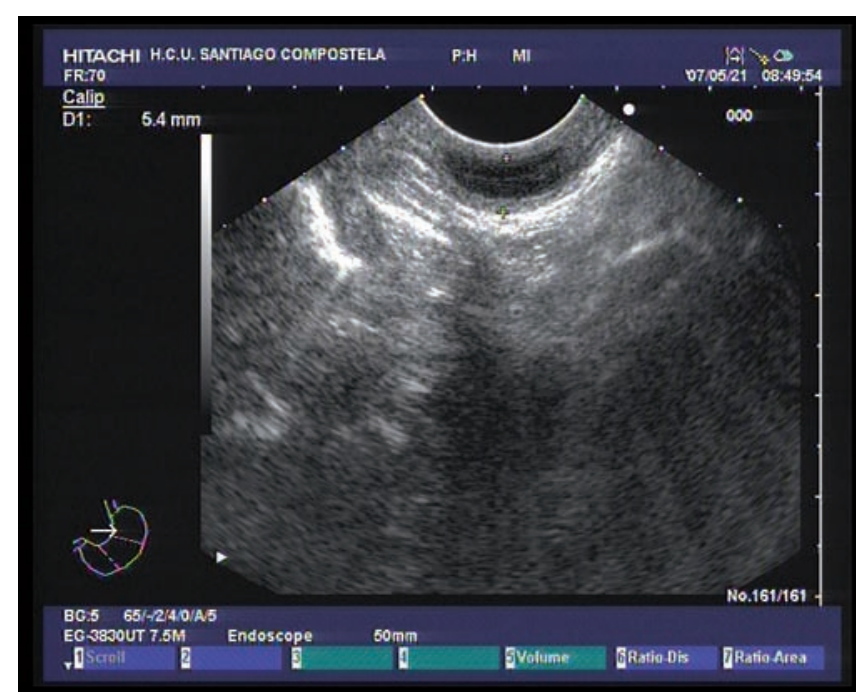

Fig. 1. Imagen por ultrasonografía endoscópica dónde se aprecia una lesión anecoica de aspecto quístico, localizada en la pared del estómago, que parece depender de la capa submucosa.

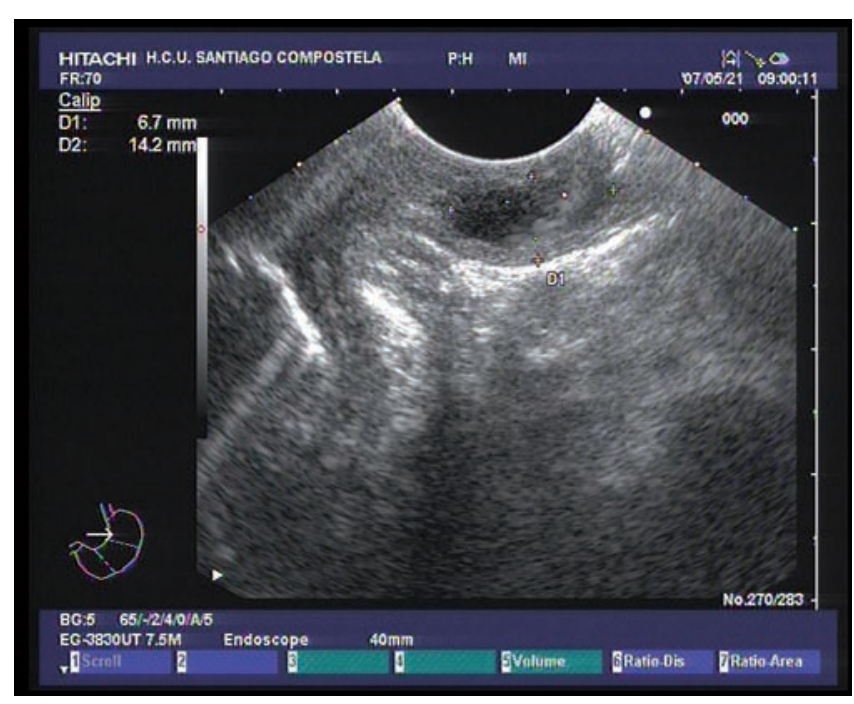

Fig. 2. Imagen por ultrasonografía endoscópica que muestra las dimensiones de la lesión quística gástrica. 
Se utiliza la tinción de Diff-Quick (Merck) para evaluar la muestra en la sala de exploraciones y se completa el estudio citológico con la tinción Papanicolau (Merck). En el examen citológico se evidencian abundantes histiocitos espumosos y escaso epitelio cilíndrico indicativo de quiste benigno compatible con quiste de duplicación gástrico (Figs. 3 y 4 ).

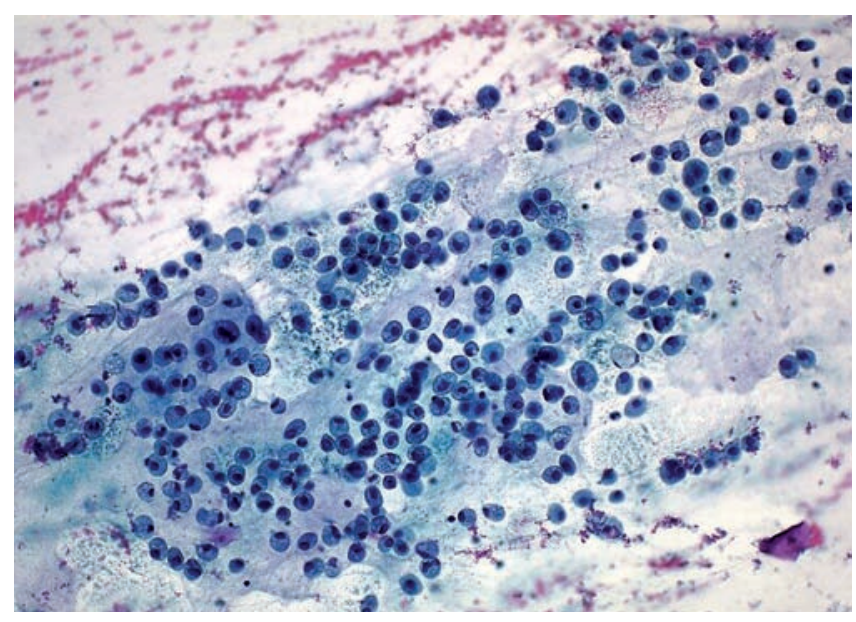

Fig. 3. Imagen panorámica de las extensiones del material aspirado con presencia de abundantes histiocitos de citoplasma espumoso (tinción de Papanicolau, 200X).

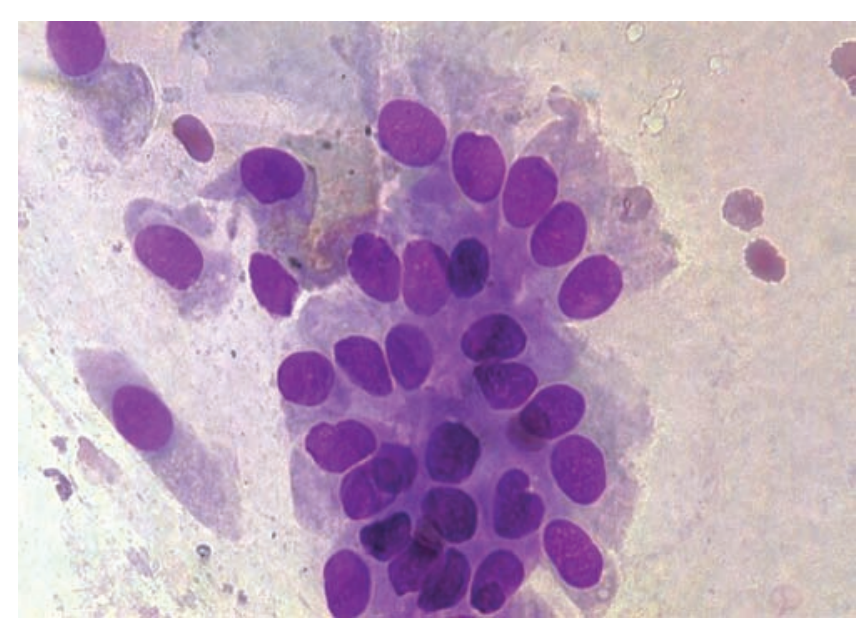

Fig. 4. A mayor aumento se observan grupos epiteliales de células cilíndricas (Diff-Quick, 400X).

\section{DISCUSIÓN}

Los quistes de duplicación son formaciones quísticas adyacentes a una porción del tracto gastrointestinal con la que comparten una pared. Se trata de tumoraciones quísticas tapizadas por mucosa, generalmente similar a la de la región anatómica adyacente. Son anomalías congénitas de muy baja prevalencia, que pueden localizarse a cualquier nivel del tracto gastrointestinal: desde la boca hasta el ano. Por orden descendente de frecuencia se localizan en ileon, esófago, yeyuno, colon, estómago y apéndice $(1,2)$. Por tanto, como acabamos de mencionar, si los quistes de duplicación gastrointestinales son poco frecuentes, más raros son aún los quistes de duplicación gástricos, que representan tan sólo entre el 2 y el $8 \%$ de todas estas entidades, según las series (1-3).

Estas estructuras son el resultado de alteraciones en el desarrollo embrionario. Se han postulado varias teorías etiopatogénicas que tratan de explicar su formación: a) persistencia de una vacuola formada en la fase sólida del desarrollo del intestino embrionario o de un divertículo embrionario; b) fallo en la fusión y recanalización de los pliegues longitudinales intestinales lo que permitiría un paso de un puente de tejido epitelial; y c) la formación de un divertículo por tracción por un fallo en el desarrollo habitual de la notocorda y endodermo que acabaría originando el quiste de duplicación. No obstante, aún no existe una teoría que explique de forma satisfactoria el desarrollo de todos lo quistes de duplicación $(1,2,4)$. Los quistes de duplicación gástricos se originan dorsalmente al intestino primitivo, por lo que la mayoría de ellos se localizan en la curvatura mayor, generalmente a nivel distal $(1,2)$ y tan sólo un $5,5 \%$ de ellos se encuentran en la curvatura menor (1).

Los quistes de duplicación afectan principalmente a mujeres, con una prevalencia de hasta del doble sobre los varones, sin observarse una tendencia familiar $(1,3,5)$. Habitualmente son diagnosticados en edad temprana, más del $60 \%$ de los casos en el primer año de vida, siendo muy raro el diagnóstico en la edad adulta $(4,6)$. Las manifestaciones clínicas dependen esencialmente de la edad del paciente, la localización y el tamaño de las lesiones. En los adultos suelen ser asintomáticos, por lo que, como sucede en nuestro caso, el diagnóstico se realiza de manera incidental en el curso de una exploración indicada por otro motivo. En nuestro caso, un varón de 39 años, se procedió al estudio ecoendoscópico de una lesión submucosa, siendo la lesión descrita un hallazgo incidental que no justificaba en ningún caso el cuadro clínico.

Las formas sintomáticas se caracterizan por dolor abdominal recurrente, masa palpable, vómitos, disminución de peso o retardo en el crecimiento. Otras manifestaciones que pueden observarse están en relación con el desarrollo de complicaciones como fiebre por sobreinfección del quiste, hemorragia digestiva, fistulización, torsión de su pedículo, o cuadros de oclusión o suboclusión intestinal $(2,3,5)$. Incluso se ha descrito el caso de un quiste de duplicación pilórico en un recién nacido simulando una hipertrofia benigna de píloro (3). En la mitad de los casos existen malformaciones asociadas, siendo las más frecuentes las duplicaciones esofágicas, seguido de anormalidades vertebrales $(1,2,5)$.

Rowling en 1959 definió una serie de características esenciales propias de los quistes de duplicación, tales como la proximidad al tracto digestivo, un aporte sanguíneo común, una capa de músculo liso que comparte con 
la pared gastrointestinal, y que se encuentre revestido por epitelio digestivo (7). Sin embargo, se han descrito casos con mucosa heterotópica como epitelio respiratorio (8) y también mucosa pancreática $(3,9)$. La presencia de mucosa pancreática y gástrica son las más asociadas a complicaciones debido a la posibilidad de desarrollo de úlcera péptica o pancreatitis aguda $(2,3)$. Se han descrito muy pocos casos de trasformación maligna, generalmente en forma de adenocarcinomas (10) y carcinomas escamosos, pero también de forma más infrecuente como tumores neuroendocrinos con diferente grado de malignidad $(11,12)$. El diagnóstico diferencial debe realizarse con neoplasias quísticas, o con degeneración quística, como el tumor mucinoso papilar intraductal, cistoadenoma mucinoso, linfangioma y con otras lesiones benignas como el quiste simple, quiste linfoepitelial, pseudoquiste pancreático, quistes de inclusión o quistes parasitarios $(4,6)$. Por ello, para la confirmación diagnóstica y especialmente para descartar la trasformación maligna del quiste es necesaria la valoración citohistológica de la lesión.

El TAC abdominal y la RMN, permiten identificar los quistes de duplicación pero los errores diagnósticos con lesiones sólidas llegan hasta un $70 \%$ según la serie publicada por Eloubeidi (13). La variabilidad de contenido de estas entidades y la presencia de un contenido proteináceo espeso en alguno de estos quistes son las principales causas de error. La USE es una técnica fundamental en el diagnóstico y caracterización de lesiones submucosas (14), permitiendo además definir con elevada precisión la relación del quiste con la pared gastrointestinal adyacente y diferenciar entre lesiones quísticas y sólidas. Hasta el momento hay nueve artículos en la literatura inglesa que mencionan de forma concreta la utilidad de la ecografía endoscópica en el diagnóstico de lo quistes de duplicación (13,15-22), incluyendo tres casos de localización gástrica $(15,20,22)$. La mayoría de autores demuestran que la USE es una técnica segura y la que presenta mayor eficacia diagnóstica para este tipo de lesiones. La USE permite además la obtención de material citohistológico, lo cual es primordial para el diagnóstico diferencial con otras entidades, así como para descartar la presencia de transformación neoplásica de los propios quistes. Aunque no existe estudio histológico en todos los casos publicados, la citología es considerada por muchos autores como un pilar fundamental en el diagnóstico de estas lesiones (13). La ecografía endoscópica muestra una mayor sensibilidad diagnóstica que la endoscopia convencional en la caracterización de lesiones submucosas del tracto gastrointestinal y la punción-aspiración con aguja fina permite una mayor eficacia en la obtención de material para estudio histológico que lo que permite la endoscopia convencional (14). Por todo ello la ecografía endoscópica, y sobre todo, la punción guiada por ultrasonografía endoscópica, se ha propuesto como la técnica de elección en el diagnóstico y caracterización de estas lesiones. La mayoría de los casos publicados muestran un tamaño de los quistes superior al nuestro, desde $2 \mathrm{~cm}$ (3) hasta $15 \mathrm{~cm}$ (11).
El hecho de que se haya practicado una punción a una lesión de características quísticas de tan pequeño tamaño podría ser tema de discusión.

Hasta el momento no existe un algoritmo diagnósticoterapéutico de este tipo de lesiones. En los casos sintomáticos se recomienda, sin ninguna discusión, la cirugía, siendo el procedimiento quirúrgico de elección controvertido. Algunos autores abogan también por la extirpación quirúrgica incluso en los casos asintomáticos, amparándose en la prevención de complicaciones y el riesgo de degeneración neoplásica $(2,6,11,23)$. Otros recomiendan un tratamiento conservador, sobre todo si tenemos en cuenta que estas lesiones en el adulto son un hallazgo incidental y que los casos descritos con transformación maligna son anecdóticos (13). Incluso se ha descrito un caso de resolución espontánea de un quiste de duplicación a nivel mediastínico (24). Zambudio y cols. proponen una serie de criterios para la escisión quirúrgica de los quistes mediastínicos: quistes sintomáticos, infección del mismo, compresión de la tráquea, crecimiento progresivo, diagnóstico en la infancia, localización o características atípicas (23). Muchos de estos criterios podrían ser extrapolados de forma razonable a los quistes de duplicación gástricos.

\section{BIBLIOGRAFÍA}

1. Kim DH, Kim JS, Nam ES, Shin HS. Foregut duplication cyst of the stomach. Pathol Int 2000; 50: 142-5.

2. O'Donnell PL, Morrow JB, Fitzgerald TL. Adult gastric duplication cysts: a case report and review of literature. Am Surg 2005; 71 (6): $522-5$.

3. Camoglio FS, Forestieri C, Zanatta C, Capelli P, Pecori S, Marcolongo A, et al. Complete pancreatic ectopia in a gastric duplication cyst: a case report and review of the literature. Eur J Pediatr Surg 2004; 14 (1): 60-2.

4. Upadhyay N, Gomez D, Button MF, Verbeke CS, Menon KV. Retroperitoneal enteric duplication cyst presenting as a pancreatic cystic lesion. A case report. JOP 2006; 7 (5): 492-5.

5. Patel MP, Meisheri IV, Waingankar VS, Ramesh S, Naregal AM, Muthaal PB. Duplication cyst of the pylorus--a rare cause of gastric outlet obstruction in the newborn. J Postgrad Med 1997; 43 (2): 43-5.

6. D'Journo XB, Moutardier V, Turrini O, Guiramand J, Lelong B, Pesenti $\mathrm{C}$, et al. Gastric duplication in an adult mimicking mucinous cystadenoma of the pancreas. J Clin Pathol 2004; 57 (11): 1215-8.

7. Rowling JT. Some observations on gastric cysts. Br J Surg 1959; 46: $441-5$.

8. Theodosopoulos T, Marinis A, Karapanos K, Vassilikostas G, Dafnios N, Samanides L, et al. Foregut duplication cysts of the stomach with respiratory epithelium. World J Gastroenterol 2007; 13 (8): 1279-81.

9. Muraoka A, Tsuruno M, Katsuno G, Sato N, Murata T, Kokudo Y, et al. A gastric duplication cyst with an aberrant pancreatic ductal system: report of a case. Surg Today 2002; 32 (6): 531-5.

10. Kuraoka K, Nakayama H, Kagawa T, Ichikawa T, Yasui W. Adenocarcinoma arising from a gastric duplication cyst with invasion to the stomach: a case report with literature review. J Clin Pathol 2004; 57 (4): 428-31.

11. Horne G, Ming-Lum C, Kirkpatrick AW, Parker RL. High-grade neuroendocrine carcinoma arising in a gastric duplication cyst: a case report with literature review. Int J Surg Pathol 2007; 15 (2): 187-91.

12. Horie H, Iwasaki I, Takahashi H. Carcinoid in a gastrointestinal duplication. J Pediatr Surg 1986; 21 (10): 902-4. 
13. Eloubeidi MA, Cohn M, Cerfolio RJ, Chhieng DC, Jhala N, Jhala D, et al. Endoscopic ultrasound-guided fine-needle aspiration in the diagnosis of foregut duplication cysts: the value of demonstrating detached ciliary tufts in cyst fluid. Cancer 2004; 102 (4): 253-8.

14. Varas MJ, Miquel JM, Abad R, Espinós JC, Cañas MA, Fabra R, et al. Interventionist endoscopic ultrasonography. A retrospective analysis of 60 procedures. Rev Esp Enferm Dig 2007; 99 (3): 138-44.

15. Hlouschek V, Domagk D, Naehrig J, Siewert JR, Domschke W. Gastric duplication cyst: a rare endosonographic finding in an adult. Scand J Gastroenterol 2005; 40 (9): 1129-31.

16. Fazel A, Moezardalan K, Varadarajulu S, Draganov P, Eloubeid MA. The utility and the safety of EUS-guided FNA in the evaluation of duplication cysts. Gastrointest Endosc 2005; 62 (4): 575-80.

17. Trojan J, Mousset S, Caspary WF, Hoepffner N. An infected esophageal duplication cyst in a patient with non-Hodgkin's lymphoma mimicking persistent disease. Dis Esophagus 2005; 18 (4): 287-9.

18. Versleijen MW, Drenth JP, Nagengast FM. A case of esophageal duplication cyst with a 13-year follow-up period. Endoscopy 2005; 37 (9): 870-2.
19. Wildi SM, Hoda RS, Fickling W, Schmulewitz N, Varadarajulu S, Roberts SS, et al. Diagnosis of benign cysts of the mediastinum: the role and risks of EUS and FNA. Gastrointest Endosc 2003; 58 (3): 362-8.

20. Ponder TB, Collins BT. Fine needle aspiration biopsy of gastric duplication cysts with endoscopic ultrasound guidance. Acta Cytol 2003; 47 (4): 571-4

21. Noguchi T, Hashimoto T, Takeno S, Wada S, Tohara K, Uchida Y. Laparoscopic resection of esophageal duplication cyst in an adult. Dis Esophagus 2003; 16 (2): 148-50.

22. Takahara T, Torigoe T, Haga H, Yoshida H, Takeshima S, Sano S. Gastric duplication cyst: evaluation by endoscopic ultrasonography and magnetic resonance imaging. J Gastroenterol 1996; 31 (3): 420-4.

23. Zambudio AR, Lanzas JT, Calvo MJ, Fernández PJ, Paricio PP. Nonneoplastic mediastinal cysts. Eur J Cardiothorac Surg 2002; 22 (5): 712-6.

24. Martin KW, Siegel MJ, Chesna E. Spontaneous resolution of mediastinal cysts. AJR Am J Roentgenol 1988; 150 (5): 1131-2. 